\title{
Embedded atom approach for gold-silicon system from ab initio molecular dynamics simulations using the force matching method
}

\author{
A NASSOUR \\ Laboratoire Sciences et Ingénierie, Matériaux et Procèdes; UMR CNRS 5266, ENSE, Grenoble, France
}

MS received 27 October 2015; accepted 15 March 2016

\begin{abstract}
In the present paper, an empirical embedded atom method (EAM) potential for gold-silicon (Au-Si) is developed by fitting to $a b$ initio force (the 'force matching' method) and experimental data. The force database is generated within $a b$ initio molecular dynamics (AIMD). The database includes liquid phase at various temperatures. Classical molecular dynamics simulations are performed to examine structural, coordination numbers, structure factors and dynamic properties of $\mathrm{Au}_{81} \mathrm{Si}_{19}$ alloy, with the interaction described via EAM model. The results are in good agreement with AIMD simulations and experimental data.
\end{abstract}

Keywords. Au-Si alloys; ab initio calculations; molecular dynamics; EAM; force matching.

\section{Introduction}

Metal-semiconductor alloy has been the subject of intense research for many years due to their potential for application in the micro-electronic devices. The gold-silicon $(\mathrm{Au}-\mathrm{Si})$ alloy is well known as a metallic glass was first obtained by rapid cooling of the liquid state [1]. Such alloy has a deep eutectic region, where the eutectic temperature $\left(359^{\circ} \mathrm{C}\right)$ is many hundreds of degrees below the melting points of $\mathrm{Au}$ $\left(1063^{\circ} \mathrm{C}\right)$ and $\mathrm{Si}\left(1414^{\circ} \mathrm{C}\right)$. It is a key system for the study of many phenomena in materials science. Among them, one can mention the recent works concerning surface induced undercooling effects [2] and surface crystallization [3]. Its technological impact is also important.

A study of physical properties such as atomic arrangements of liquid $\mathrm{Au}_{81} \mathrm{Si}_{19}$ alloy and the relation between the glassy state and the local structure of eutectic liquid has not been well-known. Recent studies, experimental and theoretical works have been devoted to investigate such properties $[4,5]$. Accurate $a b$ initio studies structural, energetic and bonding properties of amorphous were reported for $\mathrm{Au}_{x} \mathrm{Si}_{1-x}$ alloys [5]. However, the application of ab initio methods to atomistic studies of diffusion, dislocations, grain boundaries and segregation is limited; such simulations require large supercell and expensive computation. To overcome this limitation, Ercolessi and Adams [6] proposed force matching method (FMM) to create effective potential using the experimental data and $a b$ initio force calculated from molecular dynamics (MD) simulations. The FMM was applied to metallic [6], covalent [7,8], ionic systems [9] and complex intermetallic alloys [10]. A similar approach was also adopted by Brommer et al [11] and Mishin et al [12].

nassour_ayoub@yahoo.fr
Of late, Ryu and Cai [13] proposed an interaction potential within the modified embedded-atom model (MEAM) [14], the parameters of which were set to reproduce the experimental phase diagram. However, with this potential, we have not been able to reproduce correctly the properties of the liquid state, namely, the partial pair-correlation functions, the coordination numbers and the total structure factors. Dongare et al [15] developed empirical model for $\mathrm{Au}-\mathrm{Si}$ system. The potential is based on reformulation of the embedded atom method (EAM) potential [16] for metals and StillingerWeber (SW) potential [17] for silicon. Indeed, this potential is not appropriate to study $\mathrm{Au}-\mathrm{Si}$ alloy in the liquid phase via MD simulations because the three-body term is known to overstate the directional covalent bonding in liquid Si [18].

In the present work, we explore the possibility of building a reliable interatomic potential for the gold-silicon $(\mathrm{Au}-\mathrm{Si})$ alloy. To this end, we develop EAM interatomic potentials for component elements gold $(\mathrm{Au})$ and silicon $(\mathrm{Si})$ using the experimental properties and to a large number of $a b$ initio forces. The $a b$ initio database has been generated by density functional calculations using the projector-augmented wave (PAW) method within the generalized gradient approximation (GGA) for the exchange-correlation effects. Including force data from different temperatures in the liquid phase improves accuracy and transferability for a great range of simulations. Once the EAM potentials of $\mathrm{Au}$ and $\mathrm{Si}$ are obtained, the cross-potential between $\mathrm{Au}$ and $\mathrm{Si}$ atoms are determined using Johnson's [19] mixing scheme. The EAM potential proposed here can be useful to reproduce pair correlation function, coordination numbers, structure factors and dynamic properties. We consider that this EAM potential will also be an appropriate tool for the study of the interface of an $\mathrm{Au}-\mathrm{Si}$ eutectic liquid on a $\mathrm{Si}$ substrate, as well as the first stages of the growth of silicon nanowires by the vapor-liquid-solid process. 
The paper is organized as follows: in the first part, the method used to generate the EAM potentials is presented. In a second part, the procedure of the proposed MD simulations is described and the present calculations are compared with $a b$ initio MD results as well as with experimental data. Finally, a conclusion is given at the end of this paper.

\section{Construction of EAM potentials}

A general technique to illustrate atomic interactions in metals is the EAM [16]. It implicitly contains many-body interactions by a term that depends on the environment of each atom. The potential energy of a system described with the EAM method is presented as

$$
\begin{aligned}
E_{\mathrm{tot}} & =\frac{1}{2} \sum_{i j} V\left(r_{i j}\right)+\sum_{i} F\left(n_{i}\right), \\
n_{i} & =\sum_{j} \rho\left(r_{i j}\right) .
\end{aligned}
$$

Here $V\left(r_{i j}\right)$ is a pair potential and $F\left(n_{i}\right)$ is the embedding function. $\rho\left(r_{i j}\right)$ is the electron density contribution from atom $j$ to atom $i$. The total electron density $n_{i}$ at an atom position $i$ is computed via linear superposition of electron-density contribution from neighbouring atoms.

\subsection{Ab initio molecular dynamics}

The force database was generated with the Vienna $a b$ initio simulation package (VASP) [20,21], a density functional theory (DFT) code based on PAW pseudo-potentials and a plane-wave basis set. In this work, the generalized gradient approximation exchange-correlation functional of Perdew et al [22] is chosen. The valence state of each element has been defined previously in the provided PAW potentials and the plane-wave cutoff is $245 \mathrm{eV}$. The dynamical simulations were carried out in the canonical ensemble (NVT) by means of a Nosé thermostat to control temperature. Newton's equations of motion were integrated using the verlet algorithm with time step of $3 \mathrm{fs}$. A system of 256 atoms in a cubic box of length $L=17.23 \AA$ with periodic boundary conditions is considered. Because of the large cell size, the $\Gamma$-point was used for the Brillouin zone integration. For $\mathrm{Au}_{81} \mathrm{Si}_{19}$ alloy, the initial configuration was taken from the well-equilibrated liquid $\mathrm{Au}$ system in which some Au atoms were randomly substituted with $\mathrm{Si}$ atoms. For both $\mathrm{Au}$ and $\mathrm{Au}_{81} \mathrm{Si}_{19}$ systems, the volume of the cell was fixed to reproduce the experimental densities [23]. Each system is equilibrated at $T=1400 \mathrm{~K}$ for $3 \mathrm{ps}$, the run was continued for $30 \mathrm{ps}$. Then, for $\mathrm{Au}_{81} \mathrm{Si}_{19}$, the system is quenched successively to $T=1200,1000$ and $700 \mathrm{~K}$, mentioning that the same equilibration protocol at $1400 \mathrm{~K}$ was used for each temperature. The procedure is repeated in the undercooled region for $T=600,500$ and $400 \mathrm{~K}$. For pure $\mathrm{Si}$, the run has been done at $T=1750 \mathrm{~K}$, in the liquid above the melting point. For each system and temperature, 2000 configurations were used to produce averaged structural quantities such as the partial pair-correlation functions. The individual forces on all atoms at different time steps are calculated and stored. The detailed structural information of the force database is listed in table 1.

\subsection{Potentials fitted to first-principles forces and experimental data}

The coefficients in the potential are determined by minimizing the deviations between the first-principles and potential forces together with those of any other physical property that is available, that is

$$
\psi(\beta)=\psi_{\mathrm{ab}}(\beta)+\psi_{\exp }(\beta),
$$

with

$$
\begin{aligned}
\psi_{\mathrm{ab}}(\beta) & =\left(3 \sum_{k=1}^{M} N_{\mathrm{k}}\right)^{-1} \sum_{k=1}^{M} \sum_{i=1}^{N_{k}}\left|F_{k i}(\beta)-F_{k i}^{\mathrm{ref}}\right|^{2}, \\
\psi_{\exp }(\beta) & =\sum_{i=1}^{N} w_{i}\left|P_{i}(\beta)-P_{i}^{\mathrm{ref}}\right|^{2} .
\end{aligned}
$$

$\psi_{\mathrm{ab}}$ represents the contributions of the forces, $M$ is the number of atomic configuration, $N_{k}$ the number of atoms in configuration $k, F_{k i}(\beta)$ the force obtained with parameterization $\beta$ and $F_{k i}^{\text {ref }}$ the reference force from the AIMD. $\psi_{\exp }(\beta)$ contains physical properties (lattice parameters, bulk modulus, elastic constants, vibration modes, $\ldots) . P_{i}(\beta)$ are physical quantities as calculated with parameterization $\beta . P_{i}^{\text {ref }}$ is

Table 1. Configurations used in the force database.

\begin{tabular}{lccccc}
\hline System & \%Au:\%Si & Atom (Au:Si) & Configurations & Temperature (K) & r.m.s deviation (\%) \\
\hline 1 & $100: 0$ & $256: 0$ & 20 & 1000 & 15 \\
2 & $100: 0$ & $256: 0$ & 20 & 1200 & 12 \\
3 & $100: 0$ & $256: 0$ & 20 & 1400 & 10 \\
4 & $100: 0$ & $256: 0$ & 20 & 1700 & 8 \\
5 & $0: 100$ & $0: 256$ & 20 & 1300 & 16 \\
6 & $0: 100$ & $0: 256$ & 20 & 1500 & 10 \\
7 & $0: 100$ & $0: 256$ & 20 & 1700 & 12 \\
\hline
\end{tabular}

r.m.s, root-mean-square. 
the reference quantities may be obtained with the experiment or the $a b$ initio calculation. The $w_{\mathrm{i}}$ are the weights of the different terms.

The experimental part of our database includes the following physical properties of $\mathrm{Au}$ and $\mathrm{Si}$ : the cohesive energy, lattice constants, bulk modulus and elastic constants $\left(C_{\mathrm{ij}}\right)$ at $0 \mathrm{~K}$, the energy and pressure according to the universal equation of Rose at 11 different lattice spacing $\left(a / a_{0}=0.90\right.$, $0.94,0.97,1.05,1.11,1.20,1.30,1.40,1.50,1.60$ and 1.75) and two final constraints to satisfy the invariance properties of the potential $\left(n_{\text {bulk }}=1\right.$ and $F_{0}\left(n_{\text {bulk }}\right)=0$ ).

The fitting is done in the following manner: the pair potential, density and embedded functions of EAM interactions are all represented numerically by splines functions with 13 parameters. The latter are optimized using the conjugate gradient method for the minimization process. The process is repeated until the difference in the values of $\psi(\beta)$ from successive minimization converges to small values. The embedding energy functions, the electron density functions and the pair energy functions for $\mathrm{Au}$ and $\mathrm{Si}$ are plotted in figure 1.

\subsection{Bulk properties}

The analysis of the $\mathrm{Au}_{81} \mathrm{Si}_{19}$ alloy requires a preliminary step, the study of Au and Si systems. This step is essential to test the reliability of the new empirical potential that will be used later to calculate the pair correlation function, structure factor and dynamic properties of $\mathrm{Au}_{81} \mathrm{Si}_{19}$ alloy. We determined lattice parameter, elastic constants, as well as bulk modulus of the elements $\mathrm{Au}$ and $\mathrm{Si}$ in the face-centered cubic (FCC) and diamond structure, respectively. The results are shown in tables 2-3, which also contain the results of the earlier empirical models as well as the experimental data. The calculated lattice parameter of $\mathrm{Au}$ and $\mathrm{Si}$ are 4.07 and $5.430 \AA$, respectively. These values compare quite well with the experimental results of 4.07 and $5.429 \AA$, the agreement with available experimental and theoretical values for elastic constants $\left(C_{11}-C_{12}\right.$, $C_{44}$ ) and bulk modulus is also good. In addition to these parameters, we also present in tables 2 and 3, the cohesive energy. The obtained $E_{\mathrm{coh}}=-3.92$ and $-4.62 \mathrm{eV}$ atom $^{-1}$ for $\mathrm{Au}$ and $\mathrm{Si}$, respectively, are closed to the values of experimental data $-3.93(-4.63) \mathrm{eV}$ atom $^{-1}$. The overall agreement with measurement is substantially better than previous calculation. For instance, previous empirical approach parameterized by Johnson's et al [24] appears to overestimate the lattice parameter and to underestimate the elastic constants.

Selected properties of liquid silicon near the melting temperature according to the two potentials are shown in figure 2 and compared with liquid silicon by ab initio calculations. Figure 2 displays the radial distribution function (RDF) according to the two potentials. It is seen that there are small differences between the simulation results, but overall the curves are much alike. The first peaks of the RDF are in good agreement with the $a b$ initio data.

We also compared the bond angle distribution of the silicon liquid described by EAM-FMM model and $a b$ initio
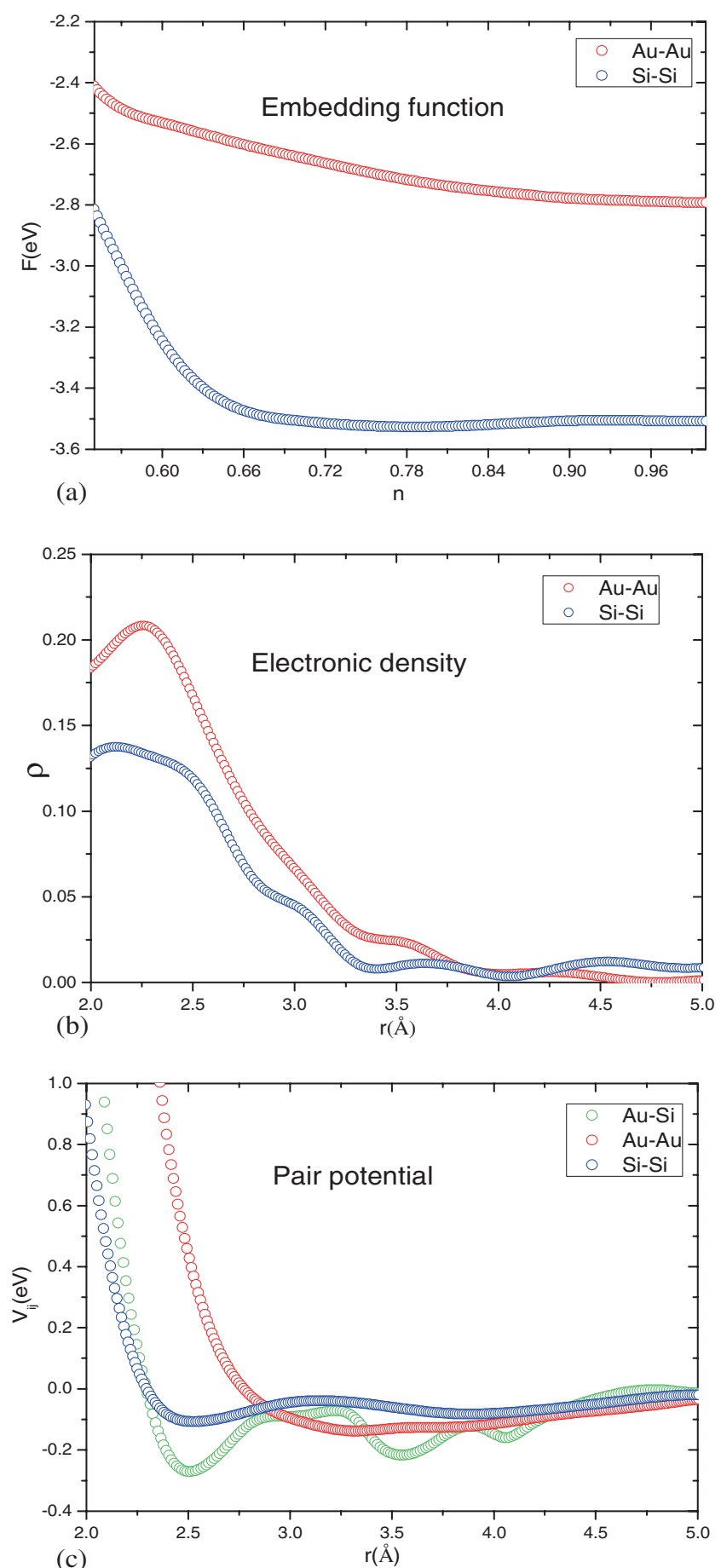

Figure 1. Plots of (a) the embedding functions for EAM-FMM $\mathrm{Au}$ (open red circles) and $\mathrm{Si}$ (open blue circles), (b) the electron density contributions for EAM-FMM potential and (c) the pair potentials. The open red circles and the open blue circles in (b) represent the electron density contribution for $\mathrm{Au}$ and $\mathrm{Si}$. The open red circles and the open blue circles in (c) correspond to the $\mathrm{Au}-\mathrm{Au}$ and $\mathrm{Si}-\mathrm{Si}$ interaction. The open green circles in (c) corresponds to the cross $\mathrm{Au}-\mathrm{Si}$ pair interaction.

simulations in figure 3. The EAM-FMM model predicts two peaks consistent with the $a b$ initio simulations and modified embedded atom method (MEAM) model [13]. It is worth 
noticing that this feature is absent in the SW model of Si [17]. The average coordination numbers from the EAM-FMM, SW [17] and ab initio method are 5.2, 4.9 and 5.5 at $1700 \mathrm{~K}$, respectively. The first minimum distance $\left(r_{\mathrm{m}}\right)$ is taken to be the first local minima of the radial distribution in our calculation. In comparison, the coordination number for the MEAM potential [13] is 5.7 at its melting temperature. Hence, the EAM-FMM model provides a reasonable description of the liquid properties of silicon.

Table 2. Calculated lattice parameters, $a_{0}(\AA)$, elastic constants, $C_{\mathrm{ij}}$ (GPA), bulk modulus, $B$ (GPA) and cohesive energy, $E_{\mathrm{coh}}$ $\left(\mathrm{eV}\right.$ atom $\left.{ }^{-1}\right)$, for $\mathrm{Au}$ from the present calculations, previous calculations and experiment.

\begin{tabular}{lcccc}
\hline Property & EAM-FMM & Johnson's $^{\mathrm{a}}$ & FBD $^{\mathrm{b}}$ & EXP $^{\mathrm{c}}$ \\
\hline$a_{0}(\mathrm{FCC})$ & 4.07 & 4.08 & 4.08 & 4.07 \\
$C_{11}-C_{12}$ & 0.317 & 0.269 & 0.245 & 0.319 \\
$C_{44}$ & 0.457 & 0.407 & 0.452 & 0.454 \\
$B$ & 1.802 & 1.700 & 1.670 & 1.803 \\
$E_{\text {coh }}$ & -3.923 & -3.930 & -3.927 & -3.930 \\
\hline
\end{tabular}

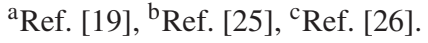

Table 3. Calculated lattice parameters, $a_{0}(\AA)$, elastic constants, $C_{\mathrm{ij}}$ (Mbar), bulk modulus, $B$ (Mbar) and cohesive energy, $E_{\mathrm{coh}}$ $\left(\mathrm{eV}\right.$ atom $\left.{ }^{-1}\right)$, for $\mathrm{Si}$ from the present calculations, previous calculations and experiment.

\begin{tabular}{lrrrr}
\hline Property & EAM-FMM & SW $^{\mathrm{a}}$ & TB $^{\mathrm{b}}$ & \multicolumn{1}{c}{ EXP } \\
\hline$a_{0}$ (dia) & 5.430 & 5.430 & 5.428 & $5.429^{\mathrm{c}}$ \\
$C_{11}-C_{12}$ & 1.008 & 0.790 & 1.008 & $1.012^{\mathrm{d}}$ \\
$C_{44}$ & 0.785 & 0.690 & 0.755 & $0.796^{\mathrm{d}}$ \\
$B$ & 0.977 & 0.980 & 1.001 & $0.978^{\mathrm{d}}$ \\
$E_{\text {coh }}$ & -4.623 & -4.630 & -4.620 & $-4.630^{\mathrm{e}}$ \\
\hline
\end{tabular}

${ }^{\mathrm{a}}$ Ref. [27], ${ }^{\mathrm{b}}$ Ref. [28], ${ }^{\mathrm{c}}$ Ref. [29], ${ }^{\mathrm{d}}$ Ref. [30], ${ }^{\mathrm{e}}$ Ref. [31].

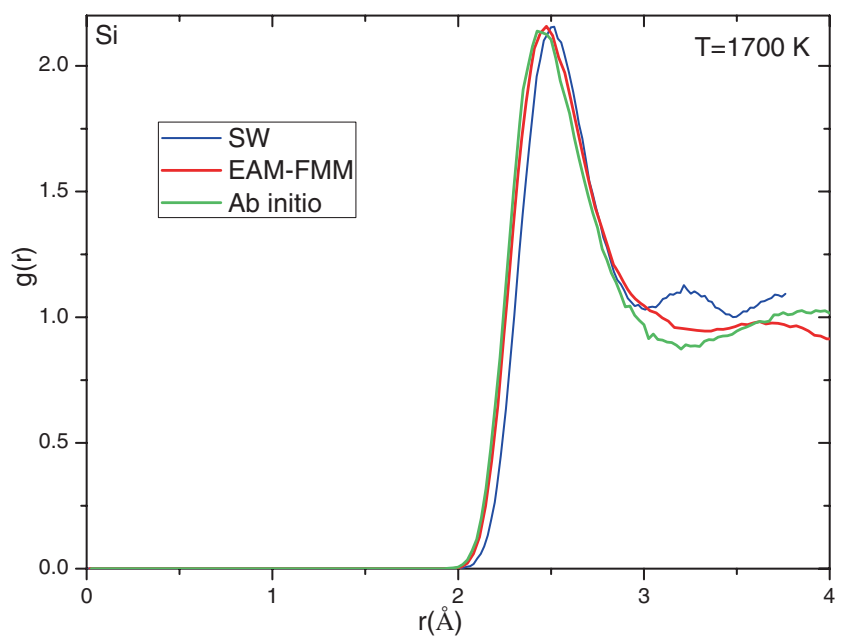

Figure 2. Pair correlation functions of liquid silicon obtained with SW, EAM-FMM and AIMD simulations.

\subsection{Cross-potential Au-Si and the fitting procedure}

The total EAM energy expression in the case of mixed pair potential involves definition of the pair potentials $V_{\mathrm{AuAu}}(r)$, $V_{\mathrm{SiSi}}(r)$ of pure-Au and $\mathrm{Si}$, the cross-potential $V_{\mathrm{AuSi}}(r)$, the electronic density functions $\rho_{\mathrm{Au}}(r), \rho_{\mathrm{Si}}(r)$ and the embedding functions $F_{\mathrm{Au}}(n), F_{\mathrm{Si}}(n)$. Therefore, the total energy can be written as follows:

$$
\begin{aligned}
E_{\mathrm{tot}}= & \frac{1}{2} \sum_{i, j \in \mathrm{Au}} V_{\mathrm{AuAu}}\left(r_{i j}\right)+\frac{1}{2} \sum_{i, j \in \mathrm{Si}} V_{\mathrm{SiSi}}\left(r_{i j}\right) \\
& +\sum_{i \in \mathrm{Au}, j \in \mathrm{Si}} V_{\mathrm{AuSi}}\left(r_{i j}\right)+\sum_{i \in \mathrm{Au}} F_{\mathrm{Au}}\left(n_{i}\right)+\sum_{i \in \mathrm{Si}} F_{\mathrm{Si}}\left(n_{i}\right) .
\end{aligned}
$$

However, we still need to build the cross-potential $V_{\mathrm{AuSi}}(r)$ and determine the scaling parameter for the relative ratio of the atomic densities $\rho_{\mathrm{Au}}(r)$ and $\rho_{\mathrm{Si}}(r)$ to obtain the best fit to alloy properties.

The cross-interaction between $\mathrm{Au}$ and $\mathrm{Si}$ is illustrated by using Johnson's mixing scheme [19]. Within this approximation, the functions $V_{\mathrm{AuAu}}(r), V_{\mathrm{SiSi}}(r), F_{\mathrm{Au}}(n), F_{\mathrm{Si}}(n), \rho_{\mathrm{Au}}(r)$ and $\rho_{\mathrm{Si}}(r)$ are taken from the Hamiltonians for the pure elements and the cross-potential pair interaction is the just new value which needs to be determined in the case of a binary alloy. This interaction is taken as

$$
V_{\mathrm{AuSi}}(r)=\frac{1}{2}\left[\frac{\rho_{\mathrm{Si}}}{\rho_{\mathrm{Au}}} V_{\mathrm{AuAu}}(r)+\frac{\rho_{\mathrm{Au}}}{\rho_{\mathrm{Si}}} V_{\mathrm{SiSi}}(r)\right] .
$$

This particular alternative of the cross-potential pair interaction guarantees invariance of the Hamiltonian under transformations given by

$$
\rho(r) \rightarrow C \rho(r), \quad F(n) \rightarrow F(n / C) .
$$

In this stage of simulations, the $C_{\mathrm{Au}} / C_{\mathrm{Si}}$ ratio allowed us to describe the $\mathrm{Au}-\mathrm{Si}$ partial pair-correlation in the liquid state as obtained from our AIMD results with reasonable accuracy. In the optimization process of fitting parameters, the relative ratio of the electron density $\rho_{\mathrm{Au}}(r)$ and $\rho_{\mathrm{Si}}(r)$ was allowed to

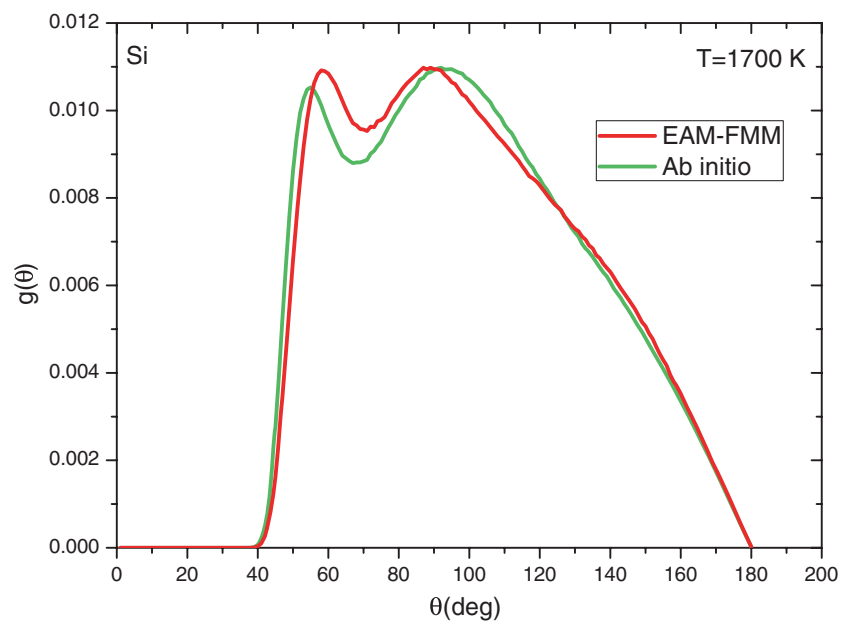

Figure 3. Bond angle distribution function of liquid silicon obtained with EAM-FMM model and AIMD simulations. 

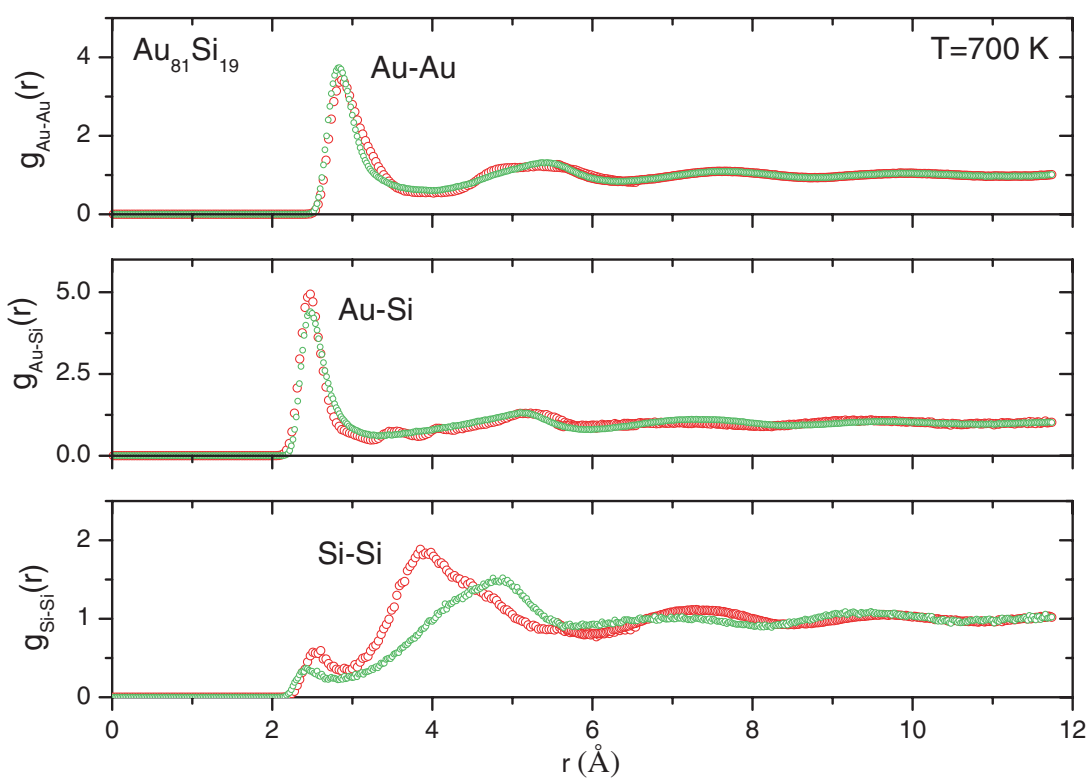

Figure 4. Partial pair-correlation functions of liquid $\mathrm{Au}_{81} \mathrm{Si}_{19}$ alloy at $T=700 \mathrm{~K}$. The open red circles correspond to classical MD with EAM-FMM potential (see text), the open green circles correspond to $a b$ initio MD results.

change. The relative ratio of the electron density $\rho_{\mathrm{Au}}(r)$ and $\rho_{\mathrm{Si}}(r)$ in the optimized potentials is 1.120 . The cross potential $\mathrm{Au}-\mathrm{Si}$ is also plotted in figure 1. This new potential is available in the tabulated form on http://www.researchgate.net/ profile/Ayoub_Nassour2 [32] or from the authors upon request.

To test the reliability of the new EAM-FMM potential, the liquid structure of the $\mathrm{Au}-\mathrm{Si}$ system is studied using classical molecular dynamics (the details of the simulation are given in the next section). As can be seen in figure 4, a comparison with the $a b$ initio curves shows a reasonable agreement of the partial pair-correlation functions of liquid $\mathrm{Au}_{81} \mathrm{Si}_{19}$ alloy at the level of the first and second peaks. From figure 4, the Au-Si bond length is the shorter one, in concordance with the first-principles simulations, the general profile of the $\mathrm{Si}-\mathrm{Si}$ partial is not in good agreement with the AIMD results.

In figure 5, the partial pair-correlation functions obtained with the novel potential are compared with AIMD simulations at $T=1400 \mathrm{~K}$ and a concentration of gold atoms $x=0.5$. While the first peak of the $\mathrm{Si}-\mathrm{Si}$ partial is a little bit underestimated, a realistic agreement is established, demonstrating some transferability of the potential to higher compositions.

\section{Results and discussion}

\subsection{Classical molecular dynamics simulations}

Using the new EAM-FMM potential, MD simulations via the LAMMPS code [33] is performed to inspect the structural and dynamic properties of liquid $\mathrm{Au}_{81} \mathrm{Si}_{19}$ alloy corresponding to the eutectic composition. For this purpose, the MD simulations are started from a super cell box with 4000 atoms under periodic boundary conditions. The temperatures are set at 400, 500, 600, 700 and $1400 \mathrm{~K}$, and experiments densities are $0.0575,0.0570,0.0565,0.0560$ and $0.0525 \AA^{-1}$. All dynamical simulations were carried out in the canonical ensemble (NVT) by means of a Nosé thermostat to control temperature. The equation of motion is solved via the velocities Verlet algorithm with a time step $1 \mathrm{fs}$. The simulation procedure is started at $T=1400 \mathrm{~K}$ from a random configuration, thermalized and cooled down at a rate of $10^{12} \mathrm{~K} \mathrm{~s}^{-1}$. At each desired temperature during cooling, a configuration is extracted and taken as an initial configuration for a new run at this temperature in the NVT ensemble. After a thermalization period of $20 \mathrm{ps}$, the run is continued for $200 \mathrm{ps}$ to produce the structural and dynamic properties.

\subsection{Structural properties}

To validate the reliability of the EAM-FMM potential and AIMD results, the structure factors of liquid $\mathrm{Au}_{81} \mathrm{Si}_{19}$ alloy are calculated. The partial structure factors $S_{\alpha \beta}(Q)$ are defined by:

$$
S_{\alpha \beta}(Q)=1+\frac{4 \pi \rho_{0}}{Q} \int_{0}^{\infty} r\left[g_{\alpha \beta}(r)-1\right] \sin (Q r) \mathrm{d} r .
$$

The positions of ions $r_{\mathrm{i}}$ are obtained by our MD simulations; $N$ is the number of ions of the $\alpha$ th species. $S_{\alpha \beta}(Q)$ are obtained by a Fourier transformation of the partial pair correlation function which is calculated directly with $a b$ initio or classical MD simulations. 

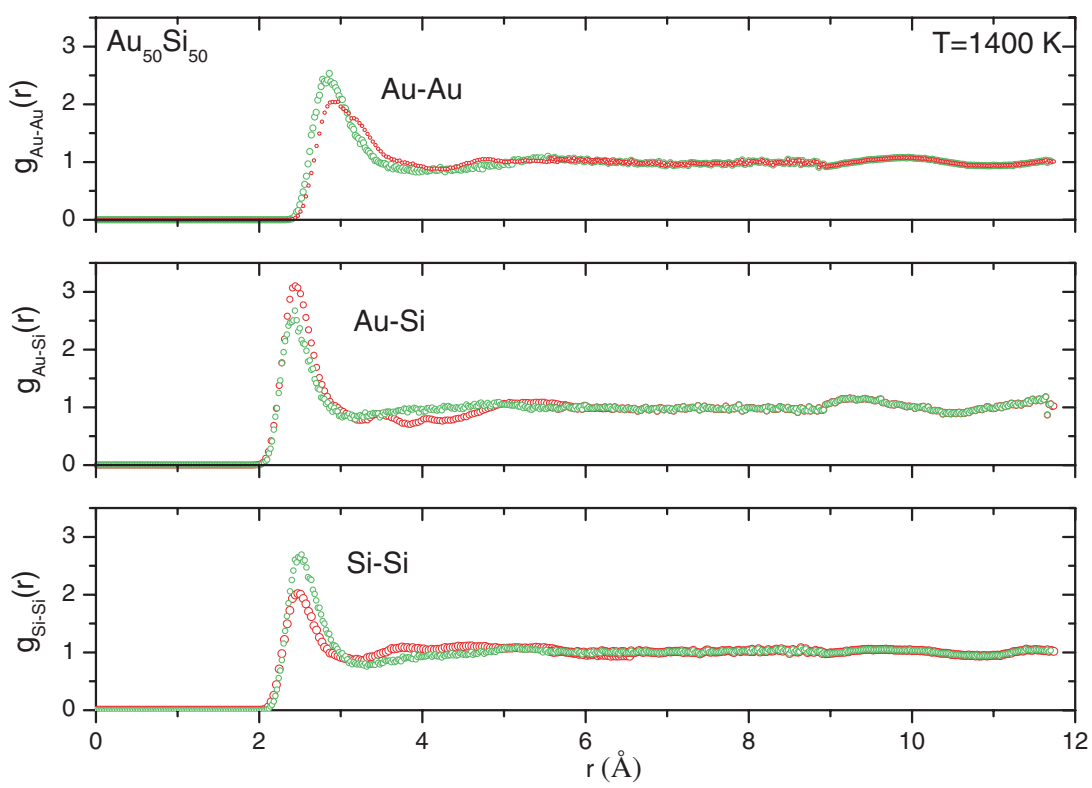

Figure 5. Partial pair-correlation functions at $T=1400 \mathrm{~K}$ for the equiatomic composition. The open red circles correspond to classical MD with EAM-FMM potential (see text), the open green circles correspond to ab initio MD results.

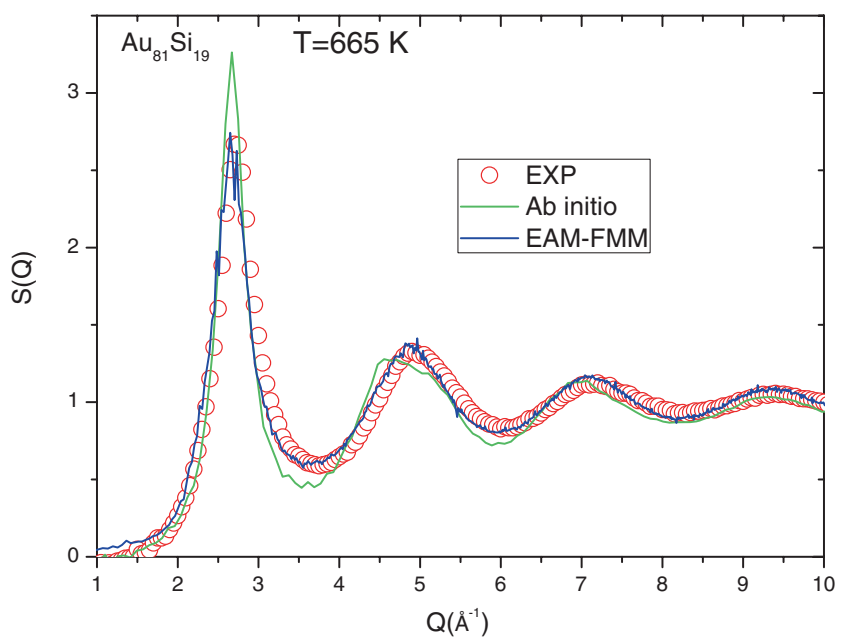

Figure 6. Structure factors for liquid $\mathrm{Au}_{81} \mathrm{Si}_{19}$ above their melting points. The open circles are the experimental data. The solid lines represent the MD simulations with the EAM-FMM potential of this work.

The total structure factor is then expressed as a linear combination of the partial structure factors $S_{\alpha \beta}(Q)$ :

$S(Q)=\sum_{\alpha} \sum_{\beta} \sqrt{c_{\alpha}} \sqrt{c_{\beta}} \frac{f_{\alpha}(Q) f_{\beta}(Q)}{c_{\alpha} f_{\alpha}^{2}(Q)+c_{\beta} f_{\beta}^{2}(Q)} S_{\alpha \beta}(Q)$,

where $f_{\alpha}(Q)$ is the atomic scattering factor of the $\alpha$ th species.

In figure 6, the total structure factors, $S(Q)$ of $\mathrm{Au}_{81} \mathrm{Si}_{19}$ alloy are displayed. It can be observed that our simulations

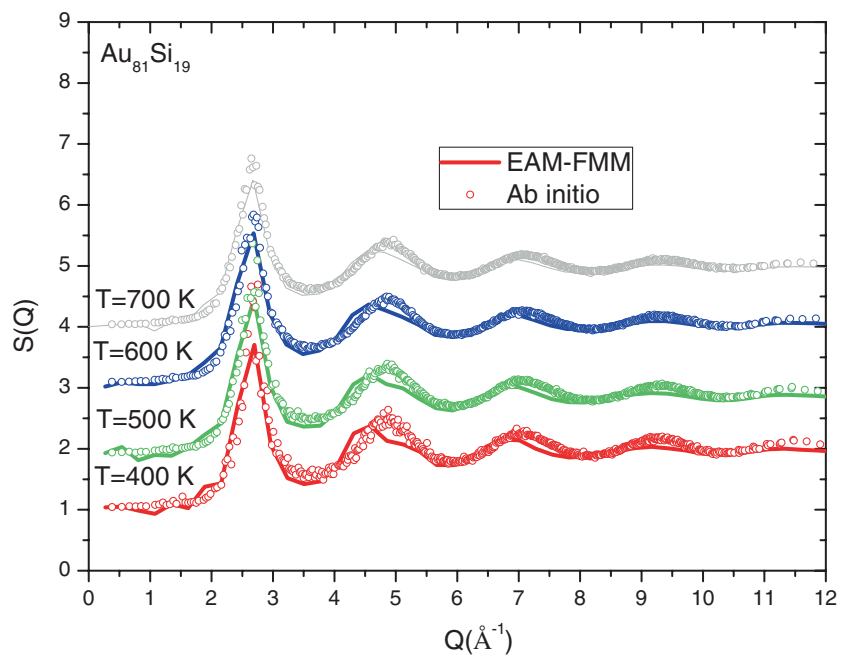

Figure 7. Calculated total structure factors, $S(Q)$ of $\mathrm{Au}_{81} \mathrm{Si}_{19}$ alloy (solid lines) as a function of temperature. The open circles are the AIMD simulations. The solid lines represent the EAM-FMM potential of this work.

agree well with experimental X-ray diffraction measurements [34] for the liquid phase. Note that our results are also in agreement with AIMD simulations.

In addition, the structure factors as a function of temperature determined from the EAM-FMM potential and AIMD results are presented in figure 7. It is seen that excellent agreement has been obtained between simulations. As it can be seen, the $S(Q)$ is quite independent of temperature change.

We have also investigated the partial pair correlation functions (PCFs) obtained from the EAM-FMM potentials for 
$\mathrm{Au}_{81} \mathrm{Si}_{19}$ alloy when the temperature is ranging from 700 to $400 \mathrm{~K}$ with respect to the AIMD simulations. The partial PCFs analyses are among the most important methods to reveal the structural features of a system, particularly for liquid and amorphous structures. These functions give the probability of finding an atom $\alpha$ at distance $r$ from an atom $\beta$ taken as the origin. To the best of our knowledge, no experimental data for partial PCFs have been reported in the literature for $\mathrm{Au}_{81} \mathrm{Si}_{19}$ alloy.

Given the partial PCFs, it is possible to estimate the partial coordination numbers (PCNs) as:

$$
N_{\alpha \beta}=\int_{0}^{r_{\min }} 4 \pi r^{2} \rho_{\alpha \beta} g_{\alpha \beta}(r) \mathrm{d} r,
$$

where $r_{\min }$ is the first minimum coordinate defined as partial PCFs, $\rho_{\beta}$ the density number of the species $\beta$. The average PCNs can provide an indication of the nature of the shortrang order.

In table 4 , the total coordination numbers as well as the coordination number around each species for $700 \mathrm{~K}$ are presented. The PCNs, given in table 4, are in better conformity with respect the AIMD results. The variations of PCNs vs. temperature are shown in figure 8. It is found that the coordination number remains constant when the temperature changes.

To test the transferability of the new potential, we study the energy and elastic properties of B2 structure of $\mathrm{Au}-\mathrm{Si}$. The results are shown in table 5, which also contains the results of the former modified embedded atom method (MEAM) model [35] as well as the $a b$ initio calculations. The calculated lattice parameter of $\mathrm{Au}-\mathrm{Si}$ is $3.226 \AA$. This value compare well with DFT calculation, the agreement with available theoretical values for elastic constant $C_{11}$ and bulk modulus is also good. Notice that the $a b$ initio model predicts $C_{44}<0$ for B2 structure, signifying that they are mechanically unstable. In comparison, the EAM-FMM and MEAM model predicts $C_{44}>0$, meaning that they may be metastable in the EAM-FMM and MEAM models. In addition to these parameters, we also present the cohesive energy in table 5. The obtained $E_{\text {coh }}=3.901 \mathrm{eV}$ for Au-Si is closed to the value of first-principles calculation $3.965 \mathrm{eV}$. Overall, the good agreement between DFT and previous MEAM model indicates the reliability of EAM-FMM potential.

Table 4. The partial coordination numbers, $N_{\alpha \beta}$ at $T=700 \mathrm{~K}$.

\begin{tabular}{lrrr}
\hline$N_{\mathrm{C}}$ & EAM-FMM & Ab initio & MEAM $^{\mathrm{a}}$ \\
\hline$N_{\text {tot }}$ & 12.25 & 12.20 & 11.75 \\
$N_{\mathrm{Au}}$ & 13.31 & 13.10 & 13.33 \\
$N_{\mathrm{Si}}$ & 7.81 & 8.30 & 5.09 \\
$N_{\mathrm{Au}-\mathrm{Au}}$ & 11.52 & 11.25 & 12.56 \\
$N_{\mathrm{Si}-\mathrm{Si}}$ & 0.14 & 0.10 & 0.22 \\
$N_{\mathrm{Au}-\mathrm{Si}}$ & 1.78 & 1.86 & 1.85 \\
$N_{\mathrm{Si}-\mathrm{Au}}$ & 7.65 & 8.20 & 3.24 \\
\hline
\end{tabular}

${ }^{\mathrm{a}}$ Ref. [36].

\subsection{Dynamic properties}

We studied the diffusion of atoms in the liquid by calculating mean-square displacement (MSD) defined in the usual way for species $\alpha$ as:

$$
\left\langle\Delta r_{\alpha}(t)^{2}\right\rangle=\frac{1}{N_{\alpha}}\left\langle\sum_{i=1}^{N_{\alpha}}\left|r_{\alpha \mathrm{i}}(t)-r_{\alpha \mathrm{i}}(0)\right|^{2}\right\rangle,
$$

where the sum goes over all $N_{\alpha}$ atoms of species $\alpha$ and the angular brackets denotes a thermal average.

The calculated results of the MSD of the various types of atoms as a function of time for temperature ranging from 700 to $400 \mathrm{~K}$ are displayed in figure 9. From the curves, two regimes can be distinguished. At short times, the motion of atoms is ballistic and the MSD is proportional to $t^{2}$. For long times, the motion is diffusive and hence, we have $\left\langle\Delta r_{\alpha}(t)^{2}\right\rangle=D_{\alpha} t+B_{\alpha}$. These regimes exist at high and low temperatures, but a third regime is often mentioned. The physical interpretation of this behaviour is so-called cage effect. At very short times, the motion of the atoms is ballistic

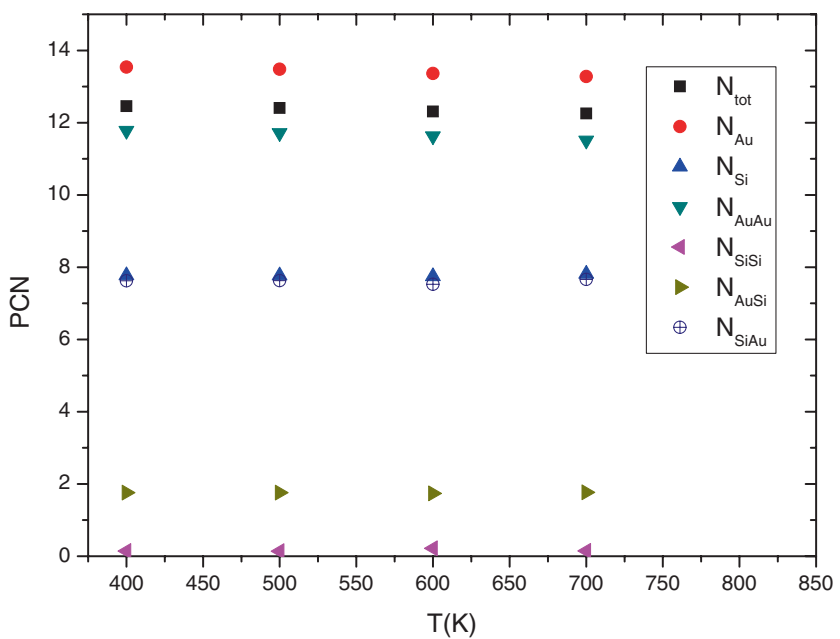

Figure 8. Partial, total and average coordination numbers of $\mathrm{Au}_{81} \mathrm{Si}_{19}$ alloy as a function of temperature obtained via $\mathrm{MD}$ with the EAM-FMM potential of this work.

Table 5. Comparison between EAM-FMM, MEAM and ab initio predictions for the energy and elastic properties of B2 structure of Au-Si. $a_{0}(\AA)$ is the equilibrium lattice constant, $B(\mathrm{GPa})$ is the bulk modulus, $E_{\mathrm{c}}(\mathrm{eV})$ is the cohesive energy and $C_{11}(\mathrm{GPa})$ and $C_{44}(\mathrm{GPa})$ are cubic elastic constants.

\begin{tabular}{lccc}
\hline Property & EAM-FMM & Ab initio & MEAM $^{\mathrm{a}}$ \\
\hline$a_{0}(\mathrm{~B} 2)$ & 3.230 & 3.225 & 3.370 \\
$C_{11}$ & 95 & 102 & 88 \\
$C_{44}$ & 0.5 & -4 & 0.7 \\
$B$ & 120 & 126 & 114 \\
$E_{\text {coh }}$ & 3.901 & 3.965 & 3.844 \\
\hline
\end{tabular}

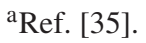



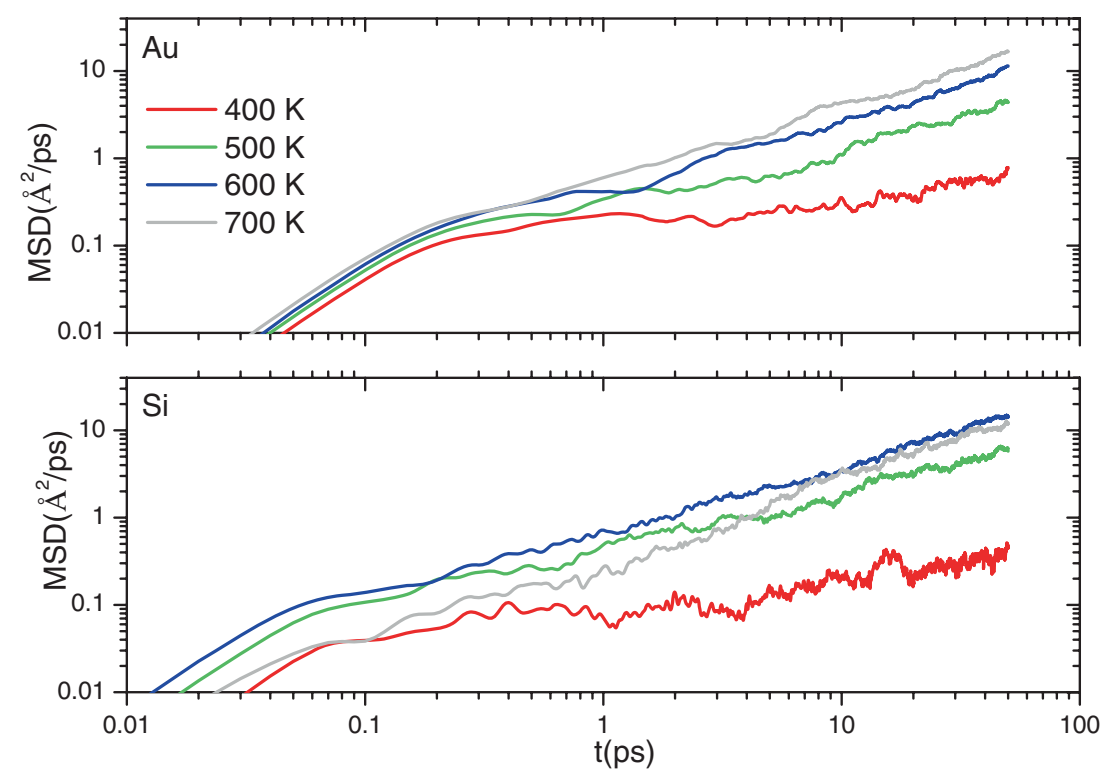

Figure 9. Mean-square displacements of $\mathrm{Au}$ and $\mathrm{Si}$ atoms calculated from MD with the EAM-FMM potential of this work, for temperatures between $T=700$ and $400 \mathrm{~K}$ at intervals of $100 \mathrm{~K}$.

Table 6. Self-diffusion coefficients obtained from MD with the EAM-FMM potential of this work and the $a b$ initio simulations, for temperatures between $T=700$ and $400 \mathrm{~K}$.

\begin{tabular}{lcccc}
\hline$D_{\text {Au }}\left(\AA^{2} \mathrm{ps}^{-1}\right)$ & $T=400 \mathrm{~K}$ & $T=500 \mathrm{~K}$ & $T=600 \mathrm{~K}$ & $T=700 \mathrm{~K}$ \\
\hline EAM-FMM & 0.002 & 0.015 & 0.036 & 0.061 \\
Ab initio & 0.004 & 0.020 & 0.041 & 0.073 \\
& & & & \\
$D_{\mathrm{Si}}\left(\AA^{2} \mathrm{ps}^{-1}\right)$ & $T=400 \mathrm{~K}$ & $T=500 \mathrm{~K}$ & $T=600 \mathrm{~K}$ & $T=700 \mathrm{~K}$ \\
\hline EAM-FMM & 0.004 & 0.019 & 0.049 & 0.073 \\
Ab initio & 0.004 & 0.020 & 0.042 & 0.064 \\
\hline
\end{tabular}

and they do not enter in collision with their nearest neighbours. For sometime, the atoms meet their nearest neighbours and hence make an escape from its cage very unlikely. Only for sufficiently large time, the atoms do succeed in escaping this cage and MSD starts again to increase significantly. The dynamics of atoms related to this cage is called modecoupling theory.

The evolution of the self-diffusion constants is also studied, because this quantity is important to understand both the amorphization phenomena and factors limiting the growth of silicon nanowires in the vapor-liquid-solid process. The self-diffusion constants $D_{\alpha}$ were extracted using Einstein's law:

$$
D_{\alpha}=\lim _{t \rightarrow \infty} \frac{\left\langle\Delta r_{\alpha}(t)^{2}\right\rangle}{6 t}
$$

Table 6 shows both the evolution of the diffusion coefficient of $\mathrm{Au}$ and $\mathrm{Si}$ in the alloy vs. of temperatures from 700 to
$400 \mathrm{~K}$. It is important to note that the two chemical species, $\mathrm{Au}$ and $\mathrm{Si}$ diffuse in the same manner in the eutectic alloy. Also, it can be observed that for the lower temperature $600 \mathrm{~K}$, the diffusion becomes very low and the system is amorphized, and metastable state is created by the high speed cooling in the simulation. A comparison is done between the values of diffusion coefficients calculated via AIMD results. EAM-FMM model reproduces the results of quality nearly comparable to the $a b$ initio MD simulations. Generally, the new parameterization developed in this work has shown to be significantly reliable for computing dynamic properties.

\section{Conclusion}

In the present work, EAM-FMM potential for Au-Si alloy is developed by fitting to $a b$ initio force and experimental data. The $a b$ initio forces are generated within AIMD in the liquid state. The structural and dynamic properties of $\mathrm{Au}-\mathrm{Si}$ eutectic alloy are investigated by means of an EAM-FMM model and AIMD simulations. The new potential is able to reproduce the structural properties, specifically, the partial paircorrelation functions, the coordination numbers and the total structure factors. A good agreement between the EAM-FMM and first-principles simulations is established for the partial pair-correlation functions as well as the total structure factors. To inspect the dynamic properties, mean-square displacement and self-diffusion are determined. Analysing the self-diffusion in $\mathrm{Au}_{81} \mathrm{Si}_{19}$ alloys revealed that $\mathrm{Si}$ and $\mathrm{Au}$ diffuse in the same manner in the eutectic alloy. The new potential can be used for large-scale atomistic simulations of segregation, fractures and $\mathrm{Au} / \mathrm{Si}$ interfaces. 


\section{Acknowledgements}

We acknowledge the CINES and IDRIS under project no. INP2227/72914 as well as PHYNUM CIMENT for computational resources. The author is gratefully acknowledged for financial support under grant no. ANR:BLAN06-3 \_138079. The author thanks Alain Pasturel for useful discussions. Special thanks are due to Noel Jakse for valuable suggestions, Saad Salah for reading the manuscript and for his comments and Philip S. Salmon for supplying neutron-diffraction data notably the total structure factors of $\mathrm{Au}_{81} \mathrm{Si}_{19}$ alloy.

\section{References}

[1] Garrett P A 1960 Nature (London) 187869

[2] Schülli T U, Daudin R, Renaud G, Vaysset A, Geaymond O and Pasturel A 2010 Nature (London) 4641137

[3] Shpyrko G, Streitel R, Balagurusamy V S K, Grigoriev A Y, Deutsch M, Ocko B M et al 2006 Science 31377

[4] Pinardi A L, Leake S J, Felici R and Robinson I K 2009 Phys. Rev. B 79045416

[5] Lee S H and Hwanga G S 2007 J. Chem. Phys. 127224710

[6] Ercolessi F and Adams A J 1994 Europhys. Lett. 26583

[7] Birner S, Kim J, Richie D A, Wilkins J W, Voter A F and Lenosky T 2001 Solid State Commun. 120279

[8] Ciobanu C V and Predescu C 2004 Phys. Rev. B 70085321

[9] Umeno Y, Kitamura T, Date K, Hayashi M and Iwasaki T 2000 Comput. Mater. 25447

[10] Brommer P, Gähler F and Mihalkov M 2007 Phil. Mag. 87 2671

[11] Brommer P and Gähler F 2007 Model. Simul. Mater. Sci. Eng. 15295

[12] Mishin Y 2004 Acta Mater. 521451

[13] Ryu S and Cai W 2010 J. Phys.: Condens. Matter 22055401

[14] Baskes M I 1992 Phys. Rev. B 462727

[15] Dongare A and Zhigilei L V 2005 L V Proc. ICCES: Int. Conf. on Computational and Experimental Engineering and Sciences (1-10 Dec. 2005, India) p. 2522
[16] Daw M S and Baskes M I 1983 Phys. Rev. Lett. 501285

[17] Stillinger F H and Weber S 1985 Phys. Rev. B 315262

[18] Stich I, Car R and Parrinello M 1991 Phys. Rev. B 444262

[19] Johnson R A 1989 Phys. Rev. B 3912554

[20] Kresse G and Hafner J 1993 Phys. Rev. B 47558

[21] Kresse G and Furthmüller J 1996 Comput. Mater. Sci. 615

[22] Perdew J, Chevary J, Vosko S, Jackson K, Pederson M, Singh D and Fiolhais C 1992 Phys. Rev. B 466671

[23] Bian X, Qin J, Qin X, Wu Y, Wang C and Thompson M 2006 Phys. Lett. A 359718

[24] Johnson R A 1988 Phys. Rev. B 373924

[25] Foiles S M, Baskes M I and Daw M S 1986 Phys. Rev. B 33 7983

[26] Pearson W 1967 A handbook of Lattice Spacings and structures of metals and alloys (Pergamon, Oxford) vol. 2, p 648

[27] Stillinger F H and Weber T A 1982 Phys. Rev. A 25978

[28] Lenosky T J, Sadigh B, Alonso E, Bulatov V V, Rubia T D, Kim J et al 2000 Model. Simul. Mater. Sci. Eng. 8825

[29] Donohue J 1974 The structure of the elements (New York: Wiley)

[30] Brewer L (unpublished) (for the cohesive energy at $0 \mathrm{~K}$ ); Moore C E, Atomic Energy Levels, Natl. Bur. Stand. (U.S.) Circ. No. 467 (U.S. GPO, Washington, D.C., 1949), vol I

[31] Numerical Data and Functional Relationships Science, edited by O Madelung, M Schulz, M Landolt-Bôrnstein, New Series, Group III, Vol. 22, pt. a (Springer-Verlag, New York, 1987); Numerical Data and Functional Relationships Science, edited by O Madelung, M Schulz and $\mathrm{H}$ Weiss, Landolt-Bôrnstein, New Series, Group III, Vol. 17, pt. a (Springer-Verlag, New York, 1982)

[32] http://www.researchgate.net/profile/Ayoub_Nassour2

[33] LAMMPS molecular dynamics software, www.cs.sandia.gov/ $\sim$ sjplimp/lammps.html

[34] Chirawatkul P, Zeidler A, Salmon P S, Takeda S, Kawakita Y, Usuki T and Fischer H E 2011 Phys. Rev. B 83014203

[35] Ryu S and Cai W 2010 J. Phys.: Condens. Matter 22055401

[36] Jakse N, Nguyen T L T and Pasturel A 2011 J. Phys.: Condens. Matter 23404205 